\section{Vesiculoscopía en el tratamiento de quistes de vesícula seminal, revisión de la literatura a propósito de un caso}

Cerda-Guerrero $\mathrm{E},{ }^{1}$ Sánchez-Solís $\mathrm{G},{ }^{2}$ Gutiérrez-Aguilera $\mathrm{E},{ }^{3}$ Cerda-Guerrero J, ${ }^{4}$ Herrera-Miranda $A^{1}$

Resumen

ANTECEDENTES: los quistes de vesícula seminal son infrecuentes. Se estima una incidencia de $0.005 \%$ y se diagnostican con mayor frecuencia de la segunda a la cuarta décadas de la vida. La mayoría de los casos se manifiesta con lesión única, aunque pueden encontrarse múltiples lesiones. La presentación puede ser congénita o adquirida; la primera se asocia con alteraciones del conducto mesonéfrico (conducto de Wolf). En dos terceras partes de los casos se ha descrito agenesia renal ipsilateral (síndrome de Zinner). La forma adquirida se asocia con causas obstructivas, quizá infecciosas.

CASO CLÍNICO: paciente masculino de 43 años de edad, que acudió a consulta por síntomas urinarios bajos, de vaciamiento, acompañados de hematoespermia, tenesmo rectal y dolor pélvico.

DISCUSIÓN: en pacientes con quistes en la vesícula seminal se han observado formas y grados variables en su manifestación clínica, algunos cursan asintomáticos y otros con síntomas urinarios obstructivos, infecciones urinarias, hematuria, hematoespermia e incluso infertilidad. Cuando los quistes son de mayor tamaño puede haber síntomas gastrointestinales: estreñimiento y tenesmo rectal. Debido a que estos quistes son infrecuentes, no existe consenso actual acerca de la conducta a seguir y el tratamiento. Hoy el tratamiento se reserva para pacientes sintomáticos; diversos artículos proponen el abordaje endoscópico, como la primera opción de tratamiento, considerando a la vesiculoscopia como el patrón de referencia para el tratamiento de los quistes de la vesícula seminal.

PALABRAS CLAVE: quiste, vesícula seminal, vesiculoscopia, hematoespermia, quistes genitourinarios.

Rev Mex Urol. 2017 March;77(2):151-157.

\section{Vesiculoscopy in the treatment of seminal vesicle cysts: A literature review in relation to a case}

Cerda-Guerrero $E,{ }^{1}$ Sánchez-Solís $\mathrm{G},{ }^{2}$ Gutiérrez-Aguilera $E,{ }^{3}$ Cerda-Guerrero $\mathrm{J},{ }^{4}$ Herrera-Miranda $\mathrm{A}^{1}$

\section{Abstract}

BACKGROUND: seminal vesicle cysts are rare entities. Their estimated incidence is $0.005 \%$ and they are more frequently diagnosed between

\footnotetext{
${ }^{1}$ Servicio de Urología, Hospital General de Occidente, Facultad de Medicina, Universidad de Guadalajara, Zapopán, Jalisco,México

${ }^{2}$ Servicio de Urología, Hospital Central Ignacio Morones Prieto, Facultad de Medicina, Universidad Autónoma de San Luis Potosí, San Luis Potosí, S.L.P, México

${ }^{3}$ Centro de Medicina Reproductiva Filius, Hospital Lomas de San Luis, Facultad de Medicina, Universidad Autónoma de San Luis Potosí, San Luis Potosí, S.L.P, México

${ }^{4}$ Facultad de Medicina, Universidad Autónoma de San Luis Potosí, San Luis Potosí, S.L.P, México
}

Recibido: diciembre 2016

Aceptado: marzo 2017

Correspondencia

Enrique Cerda Guerrero

urologiazoquipan@gmail.com

Este artículo debe citarse como

Cerda-Guerrero E, Sánchez-Solís G, GutiérrezAguilera E, Cerda-Guerrero J, Herrera-Miranda A. Vesiculoscopía en el tratamiento de quistes de vesícula seminal, revisión de la literatura a propósito de un caso. Reporte de un caso. Rev Mex Urol. 2017 mar;77(2):151-157. 
the second and fourth decades of life. The majority of cases present as a single lesion, but multiple lesions have been reported. They are congenital or acquired, and the former is associated with mesophrenic duct (Wolffian duct) alterations. Two thirds of the cases include the presence of ipsilateral renal agenesis (Zinner syndrome). The acquired form is associated with obstructive, presumably infectious, causes.

CLINICAL CASE: a 43-year-old man sought medical attention for lower urinary tract voiding symptoms, accompanied with hematospermia, rectal tenesmus, and pelvic pain.

DISCUSSION: varying forms and degrees of clinical presentation have been observed in patients with seminal vesicle cysts. Some patients are asymptomatic, whereas others present with lower urinary tract symptoms, urinary infections, hematuria, hematospermia, and even infertility. Large cysts can be accompanied with gastrointestinal symptoms, such as constipation and rectal tenesmus. Because it is a rare condition, there is no consensus on the treatment and management of these patients. At present, treatment is reserved for symptomatic patients. Different authors propose the endoscopic approach as firstline treatment, considering vesiculoscopy as the criterion standard in seminal vesicle cyst management.

KEY WORDS: seminal vesicle cyst, vesiculoscopy, hematospermia, genitourinary cysts
1 Servicio de Urología, Hospital General de Occidente, Facultad de Medicina, Universidad de Guadalajara, Zapopán, Jalisco,México

2 Servicio de Urología, Hospital Central Ignacio Morones Prieto, Facultad de Medicina, Universidad Autónoma de San Luis Potosí, San Luis Potosí, S.L.P, México

3 Centro de Medicina Reproductiva Filius, Hospital Lomas de San Luis, Facultad de Medicina, Universidad Autónoma de San Luis Potosí, San Luis Potosí, S.L.P, México

4 Facultad de Medicina, Universidad Autónoma de San Luis Potosí, San Luis Potosí, S.L.P, México

Correspondencia

Enrique Cerda Guerrero

urologiazoquipan@gmail.com

\section{ANTECEDENTES}

Los quistes de la vesícula seminal son infrecuentes; su incidencia se estima en $0.005 \% .^{1,2,3}$ Algunos autores consideran que hay 100 casos reportados en la bibliografía. ${ }^{4}$ Se diagnostican con mayor frecuencia de la segunda a la cuarta décadas de la vida, ${ }^{5,6,7}$ durante el periodo de mayor actividad sexual. ${ }^{6,7}$

Se desconoce la causa exacta de su origen, su presentación es congénita o adquirida. ${ }^{7,8}, \mathrm{La}$ primera se asocia con alteraciones del conducto mesonéfrico (conducto de Wolf). ${ }^{3-6,10,11} \mathrm{El}$ conducto mesonéfrico en el hombre da lugar a la vesícula seminal e induce el desarrollo renal a través del brote ureteral. Las malformaciones del brote ureteral pueden causar agenesia renal, displasia, hipoplasia o, incluso, duplicación de los conductos eyaculadores, obstrucción de la vesí- cula seminal y provocar la formación de quistes de la vesícula seminal, ${ }^{2-4}$ dos terceras partes de los casos se acompañan de agenesia renal ipsilateral (síndrome de Zinner). ${ }^{1,6,9}$ Se ha descrito su asociación con enfermedad poliquística renal. En los quistes bilaterales de vesícula seminal existe, incluso, $44-60 \%$ de enfermedad poliquística renal. ${ }^{9}$ La forma adquirida se asocia con causas obstructivas, presumiblemente infecciosas. ${ }^{2-6} \mathrm{La}$ mayoría de los casos se manifiesta como una lesión única, aunque pueden encontrarse varias lesiones; esto es más frecuente en las formas adquiridas. ${ }^{6}$ Los quistes de la vesícula seminal son las lesiones más infrecuentes de los quistes del aparato genitourinario masculino. Cuadro 1

\section{CASO CLÍNICO}

Paciente masculino de 43 años de edad, padre de tres hijos, con antecedente de enfermedad vené- 
Cuadro 1. Quistes genitourinarios, según su ubicación en relación con la próstata

Quistes del aparato genitourinario masculino
Intraprostáticos
Quistes mediales
Quistes del utrículo prostático
Quistes müllerianos
Quistes paramediales
Quistes del conducto eyaculador
Quistes laterales
Quistes prostáticos de retención
Degeneración quística asociada con HBP
Quistes asociados con neoplasias
Abscesos prostáticos
Quistes extraprostáticos
Quistes de vesícula seminal
Quistes del conducto deferente
Quistes del conducto de Cowper

rea en la adolescencia. Lo diagnosticó y trató un dermatólogo como papulomatosis bowenoide.

El paciente acudió a la consulta de Urología del Hospital Central Dr. Ignacio Morones Prieto por hematoespermia de dos años de evolución. Durante el interrogatorio refirió antecedentes de infecciones urinarias de repetición, dolor testicular y pélvico, y tenesmo rectal durante los últimos seis meses.

A la exploración física dirigida, los genitales se observaron acordes para su edad, pene no circuncidado, prepucio retráctil, meato uretral central, ambos testes en las bolsas escrotales, sin alteraciones evidentes, próstata hipertérmica, dolorosa, bilobulada, adenomatosa grado II, no se palparon lesiones sospechosas. El tratamiento se inició para prostatitis. El ultrasonido vesicoprostático reportó que la próstata medía 40 x $47 \times 35$ mm (Figura 1). Se trató de una lesión hipoecoica de $30 \times 23 \mathrm{~mm}$ en línea media. La lesión se corroboró en el ultrasonido prostático transrectal (Figura 2). La tomografía abdominal contrastada (Figura 3) no reportó anormalidades en la vía urinaria. En la espermatobioscopia des-

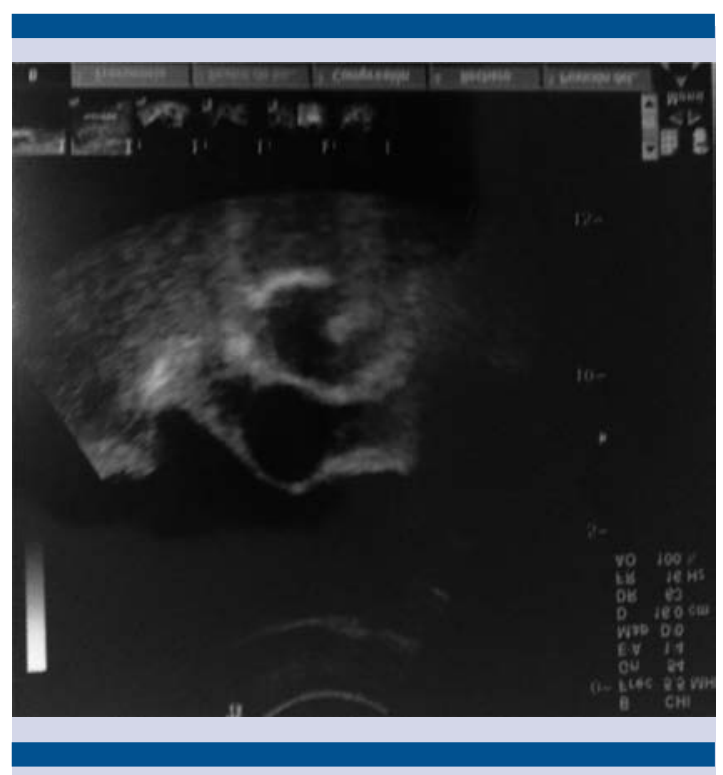

Figura 1. Imagen de un ultrasonido vésico-prostático transabdominal. Se identifica una imagen anecogénica bien delimitada en la línea media de la glándula prostática.

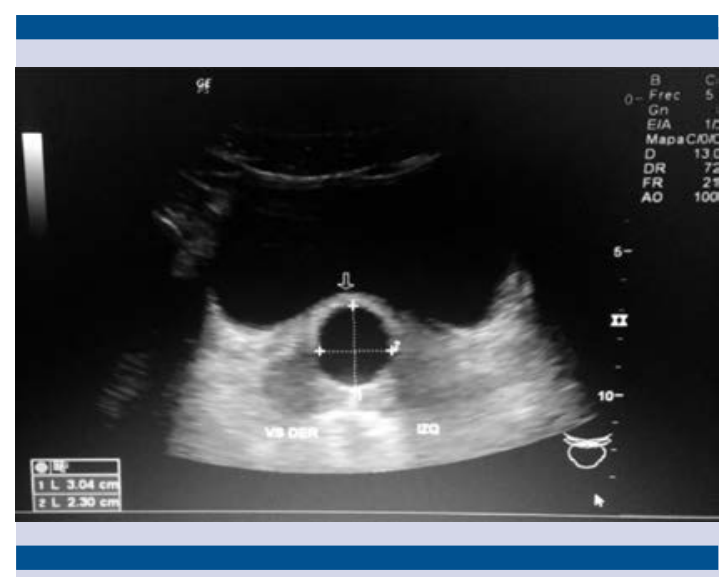

Figura 2. Imagen de ultrasonido transrectal de próstata. Se aprecia con mayor claridad la imagen anecogénica bien delimitada de 30 × 23 mm.

tacó la hematospermia. La cuenta espermática se informó con valores normales en concentración, estructura y motilidad.

Puesto que en la uretrocistoscopia no se encontraron anormalidades se procedió a la 


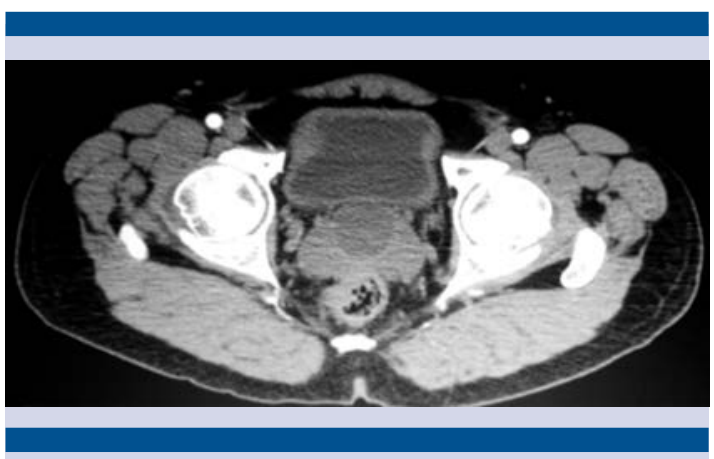

Figura 3. Imagen de tomografía axial computada abdominal, con contraste venoso. En este corte en fase arterial cerca de la próstata se aprecia una lesión homogénea hipodensa, en la línea media de la glándula prostática. En el resto del parénquima prostático no se encuentran otras lesiones. Se aprecian paredes vesicales, con discreto engrosamiento difuso, sin otras lesiones evidentes.

vesiculoscopia con un ureteroscopio pediátrico Storz F6/F7.5. Alrededor del Veru montanum, con un catéter epidural como guía, se identificaron orificios de salida del ducto eyaculador. No fue posible acceder a las vesículas seminales. El tejido periductal se resecó con un resectoscopio, con infusión con solución salina al $0.9 \%$, no mayor de $0.2 \mathrm{~L} / \mathrm{min}$. Se accedió a las vesículas seminales, se drenó material denso con contenido hemático y se realizó una infusión con solución salina y $500 \mathrm{mg}$ de levofloxacino. El procedimiento se concluyó sin encontrar otra anormalidad. Se dejó una sonda transuretral durante tres días, y se dio cita a los 30 días. En esa visita el paciente refirió disminución de los síntomas, sin hematoespermia u otro acompañante. El ultrasonido vesicoprostático de control no mostró datos de regresión. Se dará seguimientio mensual durante los siguientes cinco meses, posteriormente cada dos meses durante los siguientes 18 meses.

\section{DISCUSIÓN}

El cuadro clínico es de gran variabilidad, debido a que la mayoría de los quistes de la vesícula se- minal son pequeños (menor de $5 \mathrm{~cm})^{6,7}$ y suelen cursar asintomáticos. Los quistes grandes (mayores de 8-10 cm) casi siempre se acompañan de síntomas urinarios bajos, irritativos, infecciones urinarias, hematuria, hematospermia, infertilidad. ${ }^{6,7,12}$ Los quistes gigantes (mayores de 12 $\mathrm{cm})^{2-4}$ pueden acompañarse de síntomas gastrointestinales: estreñimiento, dolor al defecar, tenesmo rectal relacionado con la obstrucción mecánica. ${ }^{7,8}$ Se han reportado casos asociados con prostatitis crónica recurrente, epididimitis, uretritis, dolor testicular y durante la eyaculación. ${ }^{7}$

La sospecha diagnóstica es el principal elemento, la hematoespermia de etiología idiopática se acompaña de una imagen sospechosa en el ultrasonido, que ayuda a orientar el diagnóstico. El ultrasonido abdominal suele ser el primer estudio en el que se encuentran los quistes de la vesícula seminal. ${ }^{9}$

Junto con la tomografía abdominal, el ultrasonido prostático transrectal (UPTR) se considera una herramienta indispensable para tratar quistes de la vesícula seminal. ${ }^{9}$ En el ultrasonido prostático transrectal, la vesícula seminal se describe como un órgano simétrico, con forma de corbatín, posterior a la vejiga y cefálico a la próstata y con ecogenicidad discretamente inferior a la próstata. ${ }^{9} \mathrm{El}$ volumen de la vesícula seminal es de aproximadamente $14 \mathrm{~mL}$, el grosor de la pared de 1-2 mm. ${ }^{4}$ El volumen está directamente relacionado con las concentraciones de testosterona, prolactina y la abstinencia sexual. La utilidad del ultrasonido prostático transrectal se ha cuestionado en varios estudios porque se reportan $50 \%$ de falsos positivos. ${ }^{13}$ Su indicación ha tomado importancia en el tratamiento asistido por imagen; por ejemplo, en la punción quística con aguja guiada por ultrasonido. ${ }^{14}$

Los diagnósticos diferenciales a considerar son: ureterocele, dilatación de la uretra prostática posterior a la resección transuretral de próstata 
(RTUP), divertículos vesicales, hidrouréter e inserción ectópica ureteral. ${ }^{4-7}$ (Cuadro 2) Otras causas menos frecuentes que pueden mimetizar los quistes de la vesícula seminal son: quistes müllerianos, quistes del utrículo prostático, quistes del conducto deferente, quistes hidatídicos. ${ }^{2,4,15}$

La tomografía axial computada es el estudio de elección, como paso inicial del tratamiento de los quistes de la vía genitourinaria masculina; algunos autores lo consideran el de mayor utilidad para el diagnóstico y para decidir el esquema de tratamiento. ${ }^{7,10,12}$ La densidad del contenido en los quistes de la vesícula seminal es cercana a la del agua, casi siempre de características heterogéneas debido al contenido hemático; pueden encontrarse imágenes hiperecogénicas compatibles con litos. ${ }^{7-12}$

La resonancia magnética es el estudio de gabinete de mayor utilidad en el estudio de los quistes del aparato genitourinario, se reserva para pacientes en quienes no es posible realizar la tomografía abdominal contrastada. La principal desventaja es el costo del estudio. ${ }^{2-10}$

Los pacientes asintomáticos pueden "tratarse" solo con observación; ${ }^{4}$ el tratamiento se reserva para pacientes sintomáticos. ${ }^{6}$ Existente diversas modalidades de tratamiento: resección abierta, ${ }^{3}$ laparoscópica, ${ }^{3}$ asistida por robot, ${ }^{16}$ aspiración con aguja fina guiada por ultrasonido,,$^{14}$ transuretral de conducto eyaculador, ${ }^{13-17}$ vesiculoscopia

Cuadro 2. Diagnósticos diferenciales más frecuentes a establecer, en un paciente con sospecha de quiste de vesícula seminal.

Diagnósticos diferenciales

Ureterocele

Divertículo vesical

Hidrouréter por inserción ectópica

Defecto post RTUP

Quiste hidatídico con o sin destechamiento de paredes quísticas. ${ }^{13,17,18,19}$

Las primeras descripciones del tratamiento quirúrgico abierto son de 1914, por parte de Zinner. ${ }^{16}$ Debido a la localización de las vesículas seminales, el acceso abierto condiciona alto riesgo de sangrado, lesión rectal y, por la disección amplia necesaria, importante afectación de la vasculatura ureteral.

Según algunas series la aparición de urinomas es relativamente frecuente. El acceso laparoscópico con seis trócares se acompaña de menores complicaciones posquirúrgicas, con mejores resultados respecto al acceso abierto, en cuanto a días de estancia hospitalaria, dolor posquirúrgico, frecuencia de complicaciones, como urinomas, entre otros. ${ }^{16}$ La punción con aguja fina (17 gauge) guiada por ultrasonido, tanto en el acceso transrectal como transperineal, se ha asociado con altas tasas de recurrencia; por eso en la actualidad se ha abandonado su uso. ${ }^{14-18}$ En la actualidad, el acceso endoscópico es la primera opción de tratamiento, y la vesiculoscopia el patrón de referencia para quistes de la vesícula seminal. Enseguida se describirán con mayor detalle las dos modalidades en la actualidad más aceptadas.

La resección transuretral de los conductos eyaculadores, RTU-DE ó TURED por sus siglas en inglés, implica la destrucción de una estructura anatómica, el colícula seminal, y el ducto eyaculador. ${ }^{13}$ Las complicaciones asociadas comprenden: eyaculación retrógrada, flujo urinario al conducto eyaculador, incontinencia urinaria, lesión al recto, fístulas uretrales. ${ }^{13}$ En la actualidad la resección ureteral (RTU-DE) está en desuso por su riesgo inherente de eyaculación retrógrada, y solo se reserva para casos en los que no es posible accesar a la vesícula seminal debido a que los ductos eyaculadores no se pueden visualizar o están obstruidos. ${ }^{13-19}$ 
Requiere grado de habilidad considerable, su ejecución incorrecta se asocia con daño a la vesícula seminal, colículo seminal y uretra. ${ }^{13-20}$ El sangrado excesivo en el posquirúrgico afecta los resultados de la cirugía, con episodios de epididimitis asociada con reflujo urinario. ${ }^{13} \mathrm{Al}$ gunos autores consideran el uso de guías Zebra (nitinol) para mejorar la visualización y controlar la entrada a las cavidades de la vesícula seminal. ${ }^{13,17,18,19}$ Para otros autores, el diámetro y las propiedades del catéter peridural disminuyen el traumatismo a la mucosa de la vesícula seminal y se acompañan de mejores resultados. ${ }^{2}$ Debido al diámetro normal de $1.7 \pm 0.3 \mathrm{~mm}$ del ducto eyaculador ${ }^{19}$ el equipo que ha demostrado mejores resultados en las vesiculoscopias es el ureteroscopio pediátrico 6-7.5F. ${ }^{13-20}$ Sin embargo, existen grandes variaciones a lo largo del trayecto del ducto eyaculador que predisponen a la obstrucción y falta de paso del equipo. ${ }^{19}$

Estas variantes anatómicas que impiden el paso del ureteroscopio pueden favorecer la obstrucción del ducto eyaculador y condicionar la formación de los quistes de la vesícula seminal. El flujo en la vesiculoscopia debe ser tan bajo como sea posible, la presión excesiva puede destruir el epitelio de la vesícula seminal y condicionar un pronóstico desfavorable. ${ }^{13}$ Una presión de $200 \mathrm{mmHg}$ y velocidad de flujo de $0.2 \mathrm{~L} / \mathrm{min}$ se considera ideal. ${ }^{20} \mathrm{Se}$ han reportado como hallazgos frecuentes: la liberación de líquido turbio de contenido hemático y litos en el interior de la vesícula seminal. ${ }^{19}$

Para evitar el reflujo urinario con la entrada a cavidad vesical debe colocarse una sonda transuretral y mantener la derivación durante los primeros 3-5 días. ${ }^{20}$ Puesto que se trata de un padecimiento poco frecuente no se ha determinado un periodo de seguimiento ideal; algunos autores sugieren un seguimiento semanal durante el primer mes, después mensual durante los primeros seis meses, y por último, cada dos meses hasta completar dos años. ${ }^{19,20}$
La mayoría de los autores ha reportado éxito cercano al $100 \%$ con el acceso endoscópico, ${ }^{17-20}$ con regresión de los síntomas y resultados normales en las espermatobioscopias a los seis meses de la cirugía. Se han reportado casos anecdóticos de fertilidad, aunque la naturaleza multifactorial de esta última vuelve difícil la asociación directa entre tratamiento y altas tasas de concepción. ${ }^{20}$ Según algunas series el dolor posquirúrgico es el último síntoma en desaparecer. ${ }^{17}$

Fuente de financiamiento: no se recibió financiamiento de ningún tipo para poder concluir este artículo.

Conflicto de intereses: Los autores declaran no existir conflicto de intereses.

Agradecimiento: A mi esposa y a mi hija por ser la razón y la fuerza para seguir creciendo, a mis padres por ser la razón y el cómo, a mis maestros por ser un modelo de vida.

\section{REFERENCIAS}

1. Ates Y, Kilcier G, Bedir S, et al. Large Vesicula Seminalis Cyst; A very rare cause of constipation and male infertility, Kaohsiung. J Med Sci 2007; 23(6):318-320.

2. Cerruto $M$, Novella $G$, Zecchinni S, et al Use of transperineal fine needle aspiration of seminal vesicles to retrieve sperm in a man with obstructive azoospermia. Fertility and Sterility 2006;86(6):1764.e7-1764.e10.

3. Cheng G, Liu B, Xu A, et al. A Novel Surgical management for male infertility secondary to midline prostatic cyst. BMC Urology 2015;15:18.

4. El-Assmy A, Abou M. Giant Seminal Vesicle Cyst Causing Ipsilateral Hydronephrosis With Contralateral Renal Agenesis. Images in Clinical Urology 2012;79:e17-e18.

5. Ercil H, Gurlen G, Sener N, et al.A Rare Cause of lower urinary tract symptoms: retrovesical hydatid cyst. J. Pak Med Assoc. 1087-1089

6. Guo S, Xie D, He X, et al. The application of pediatric Ureteroscope for seminal vesiculoscopy. Minimally Invasive Surgery 2015;946147.e1-946147.e4

7. Haddock P, Wagner J. Seminal Vesicle Cyst with Ipsilateral Renal Agenesis and Ectopic Ureter (Zinner Syndrome). Images in Clinical Urology 2015;85:e41-e42. 
8. Han C, Liang Q, Dong B, et al. The Transurethral vesiculoscopy in the Diagnosis and Treatment of the Seminal Vesicle Disease. Cell Biochem Biophys 2013;66:851-853.

9. Heller M, Hartman M, McGreevy B. American Journal of Emergency Medicine 2012;30: 2087.e1-2087.e6

10. Kao C, Wu C, Sun G, et al. Congenital Seminal Vesicle Cyst Associated with Ipsilateral Renal Agenesis Mimicking Bladder Outlet Obstruction: A case Report and Review of the Literature, Kaohsiung. J Med Sci 2010; 26(26):30-34.

11. Li B, Zhang C, Li K, et al Clinical Analysis of the characterization of magnetic resonance imaging in 102 cases of refractory haematospermia. Andrology 2013;948-956.

12. Lin J, Wu H, Wang J, et al. Ectopic Opening of cyst Dilatation of the Ejaculatory Duct into Enlarged Prostatic Utricle. Journal of Andrology 2012;33:574-578.

13. Li Y, Liang P, Sun Z, et al. Imaging Diagnosis, Transurethral Endoscopic Observation, and Management of 43 Cases of persistent and Refractory Hematospermia. Journal of Andrology 2012;33:906-917.

14. Lotti F, Maggi M. Ultrasound of the male genital tract in relation to male reproductive health. Human Reproduction Update 2014;0:1-28.
15. Luo J, Chen W, Guo Y, et al. Images in clinical Urology 2009;503-504.

16. Shebel H, Farg $\mathrm{H}$, Kolokythas $\mathrm{O}$, et al. Cysts of the Lower Male Genitourinary Tract: Embryologic and Anatomic Considerations and Differential Diagnosis. Radiographics 2013;33:1125-1143.

17. Arora S, Breiman R, Webb E, et al. CT an MRI of Congenital Anomalies of the Seminal Vesicles. AJR 2007;130-135.

18. Scarcia M, Maselli F, Cardo G, et al. Robot Assisted excision of the seminal vesicle cyst associated with ipsilateral renal agenesis. Archivo Italiano di Urología e Andrología 2015; 325-327.

19. Wang $\mathrm{H}, \mathrm{Ye} \mathrm{H}, \mathrm{Xu} \mathrm{C}$, et al. Transurethral Seminal Vesiculoscopy Using 6F Vesiculoscope for Ejaculatory Duct Obstruction: Initial Experience. Journal of Andrology 2012;33:637-643.

20. Zhang D, Li X, Gao Y. et al. Transperitoneal Laparoscopic Excision of Seminal Vesicle Cyst: A Single-Center Experience. Journal of Endourology 2012; 26:1153-1158.

\section{AVISO PARA LOS AUTORES}

Revista Mexicana de Urología tiene una nueva plataforma de gestión para envío de artículos. En: https://www.revisionporpares.com/index.php/RMUrol podrá inscribirse en nuestra base de datos administrada por el sistema Open Journal System (OJS) que ofrece las siguientes ventajas para los autores:

- Subir sus artículos directamente al sistema.

- Conocer, en cualquier momento, el estado de los artículos enviados, es decir, si ya fueron asignados a un revisor, aceptados con o sin cambios, o rechazados.

- Participar en el proceso editorial corrigiendo y modificando sus artículos hasta su aceptación final. 